\title{
A FUZZY LOGIC BASED EXPERT SYSTEM FOR DETERMINATION OF HEALTH RISK LEVEL OF PATIENT
}

\author{
Monish Kumar Choudhury ${ }^{1}$, Neelanjana Baruah ${ }^{2}$ \\ ${ }^{1}$ Student M.E. Electrical Engineering, Jorhat Engineering College, Assam, India \\ ${ }^{2}$ Department of Electrical Engineering, Jorhat Engineering College, Assam, India
}

\begin{abstract}
The aim of this study is to design a fuzzy expert system for calculating the health risk level of a patient. The fuzzy logic system is a simple, rule-based system and can be used to monitor biological systems that would be difficult or impossible to model with simple, linear mathematics. The designed system is based on the modified early warning score (MEWS).The system has 5 input field and 1 output field. The input fields are blood pressure, pulse rate, SPO2 (it is an estimation of the oxygen saturation level in blood. ), temperature, and blood sugar. The output field refers the risk level of the patient. The output ranges from 0 to 14. This system uses Mamdani inference method. A larger value of output refers to greater degree of illness of the patient. This paper describes research results in the development of a fuzzy driven system to determine the risk levels of health for the patients. The implementation and simulation of the system is done using MATLAB fuzzy tool box.
\end{abstract}

Keywords: Fuzzy logic, The Modified Early Warning Score (MEWS), Physiological Parameters, Classification of Vital Signs, MATLAB Tool, Fuzzy Inference System, Fuzzification, Defuzzification

\section{INTRODUCTION}

In day to day life there are many situations in which it is very useful if we can determine the risk level of patients. For example occurrence of a natural digester like flood, earthquake etc. affects people in many fields of their life including their health. In such situations many people die because common people around them cannot predict that they require immediate treatment and also it became very difficult for the doctors to reach them. So if there is a system that can predict the health status of the people depending upon their physiological parameters without the help of a doctor then it will be very helpful for saving the life of many people. Motivated by the need of such an important system, in this study an expert system is designed to determine the risk level of patient so as to predict their health status. Now risk level calculation using the physiological parameters involves lots of inaccuracy and uncertainty. In such situation fuzzy logic can give much satisfactory result since it can provide accurate information when there is inaccuracy [1].The expert system is designed using Fuzzy Logic. In this work, the fuzzy logic is based on the modified early warning score (MEWS), which is a simple guide used by hospital nursing and medical staff as well as emergency medical services to quickly determine the degree of illness of a patient [2]. This fuzzy control system has been implemented in MATLAB Tool. In this paper introduced fuzzy control system to design fuzzy rule base to analyze the risk level of patient health and the rule viewed by surface view.

\section{THE FUZZY LOGIC SYSTEM}

Fuzzy Logic provides an effective tool for describing the characteristics of a system that is too complex or ill-defined to admit precise mathematical analysis. This theory is based on approximate reasoning which plays a major role in human thought process. Fuzzy logic is an Artificial Intelligence technique which has the ability to mimic human mind in terms of approximate reasoning rather than being exact [3]. A Fuzzy Set has values with partial membership along with the crisp values. Fuzzy Sets are useful in establishing conditions which are imprecise in definition through partial membership values. Elements in fuzzy set can overlap, so a given crisp value can belong to multiple fuzzy sets with different membership degrees in each set[4]. To utilize fuzzy logic, four components are required: fuzzification, an inference, a fuzzy rule base, and defuzzification [5]. One of the basic principles of fuzzy logic is the degree of membership determined by "fuzzifying" each data point using the input fuzzy set. The input fuzzy set is determined by the system designer to break down the complete range of possible input values into membership functions. Each membership function has a value of either 0 or 1 and a minimum and maximum range of input value. Several shapes for the membership function can be used, including trapezoidal, Gaussian, and triangular. The most common and simplest to understand are trapezoidal and triangular shaped membership functions, which can be assembled into a fuzzy set by setting the minimum input value of each function to the center point of the previous membership function. 


\section{MODIFIED EARLY WARNING SCORE}

\section{(MEWS)}

Early warning scoring tools are used to aid recognition of deteriorating patients, and are based on physiological parameters, which are taken when recording patient observations. The observations incorporated in this scoring system should include: temperature, pulse, blood pressure and respiratory rate, with oxygen saturations, level of consciousness and urine output. An aggregated score is then calculated from all seven parameters. There is an identified threshold score which, when reached, then activates an escalation pathway. The escalation pathway outlines actions required for timely review ensuring appropriate interventions for patients. It should be remembered that MEWS is an aid to good clinical judgment, not a substitute for it.[6] It can be used to quickly identify patients who are clinically failing and who need urgent intervention. MEWS can be used to monitor medical patients during assessment and transport. The use of MEWS has been shown to be effective in reducing death rates and illness chances of patients whose health slowly worsens. MEWS can be used to monitor medical patients during assessment and transport. The use of MEWS has been shown to be effective in reducing death rates and illness chances of patient's health.

Table 1: The Modified Early Warning Score [7]

\begin{tabular}{|l|l|l|l|l|l|l|l|}
\hline MEWS & +3 & +2 & +1 & 0 & +1 & +2 \\
\hline $\begin{array}{l}\text { Systolic blood } \\
\text { pressure }\end{array}$ & $<70$ & $70-80$ & $81-100$ & $101-199$ & & $\geq 200$ \\
\hline Heart rate & & $<40$ & $41-50$ & $51-100$ & $101-110$ & $111-130$ & $>130$ \\
\hline $\begin{array}{l}\text { Respiratory } \\
\text { rate }\end{array}$ & $<9$ & & $9-14$ & $15-20$ & $21-29$ & $\geq 30$ \\
\hline Temperature & & $<35$ & & & & & \\
\hline $\begin{array}{l}\text { AVPU/GCS } \\
\text { score }\end{array}$ & 99 & 14 & A/15 & v/confused & P & U \\
\hline AVPU=Alert, Verbal, Pain, Unresponsive; GCS=Glasgow Coma Scale & & & \\
\hline
\end{tabular}

A MEWS is calculated for a patient using the five simple physiological parameters shown in Table 1. Respiratory rate, heart rate, systolic blood pressure, temperature and AVPU. A score is given to a specific range of values for each of the parameters in the table. The patient's data for each parameter is cross referenced against the MEWS table and a score from 0 to 3 is allocated. The score for each parameter is then added to give the MEWS score. A score of zero shows that the patient case is normal, a score that is more than zero and less than five shows that the patient is in a Low Risk case, and a score of five or more shows that the patient is in a High Risk case, and an admission to an intensive care unit is recommended. In this work, different MEWS parameters were used in order to calculate the MEWS score. The parameters used are: systolic blood pressure (SBP), heart rate (HR), oxygen saturation (SPO2), body temperature (TEMP), and blood sugar (BS) .An expert's knowledge was used for dividing the input fields.

\section{FUZZY EXPERT SYSTEM DESIGNING}

MATLAB fuzzy tool box is used for implementation and simulation of the fuzzy expert system. In order to design a fuzzy expert system the typical steps followed are determination of the input and output variables, the selection of suitable membership functions, and the creation of the fuzzy rules database [8]. FIS editor used for defining the input and output variables is shown in Figure.1. Again the membership function editor used for defining the membership functions of each input and output variable is shown in Figure.2.

\subsection{Input Variables}

For designing the expert system five input variables i.e. blood pressure, heart rate, SPO2, temperature and blood sugar are used. These inputs are called vital signs and use to predict the health status of person. After choosing the input variables the next step is to fuzzify the variables i.e. we have to determine the fuzzy sets for each input variable and the corresponding range of the belonging to each fuzzy set.

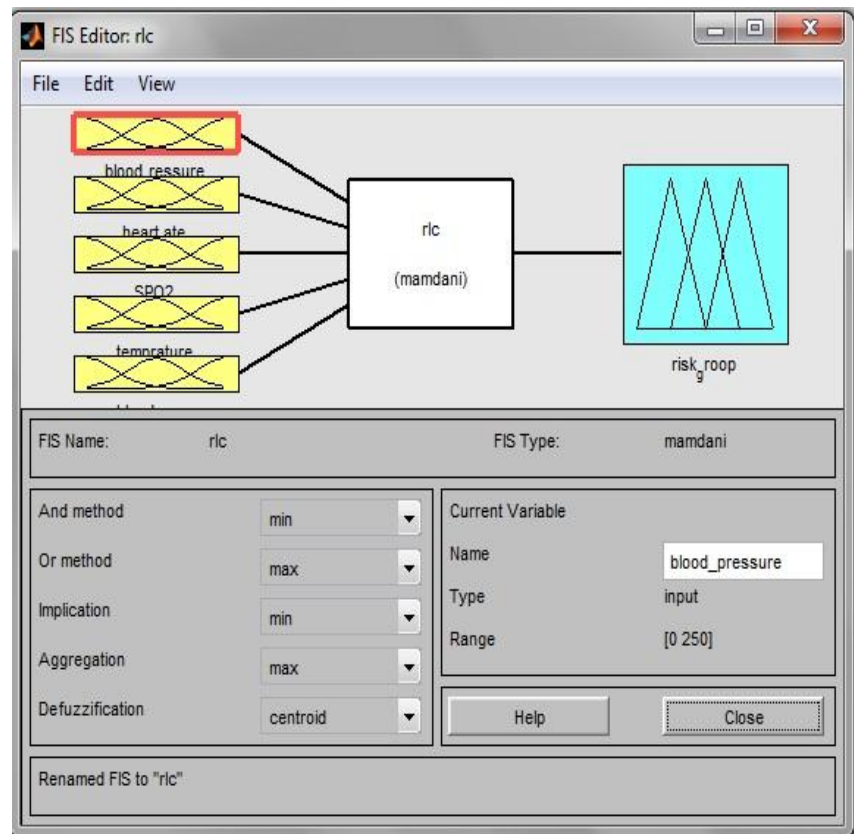

Fig. 1: Mamdani FIS editor with 5 inputs \& 1 output 


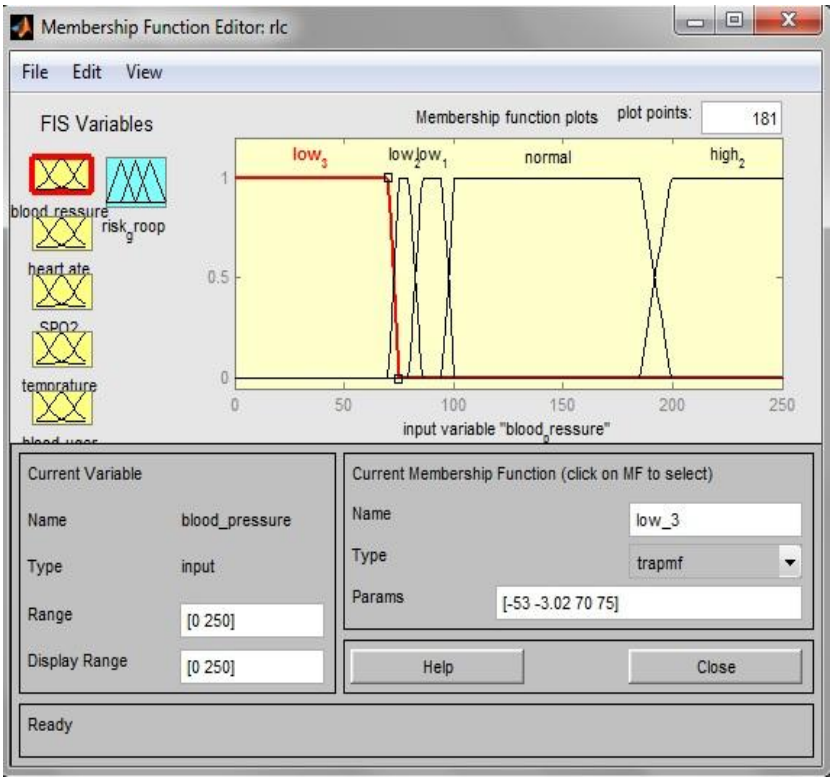

Fig. 2: Membership function editor

Depending on the MEWS scoring system and using the expert advice fuzzy sets for each of input variables are determined. Membership functions of the fuzzy sets are taken as trapezoidal. For blood pressure we use only the systolic value. To fuzzify the SBP variable, range of values for SBP which would be considered as normal are needed. Let this be 100 to $185 \mathrm{~mm} \mathrm{Hg}$ (not everyone might agree with this, so this choice merely captures the experience of one particular expert). Thus a fuzzy set labeled Normal-0 is created and values of SBP between 101 and $199 \mathrm{~mm} \mathrm{Hg}$ to a membership level of 1.0 is assigned to this set. Next we address the more vague issue of what range of values for SBP could possibly be normal but also be abnormal. Per the expert advice, the range 185 to 199 was decided to be at the upper end and 95 to 100 at the first lower end. In other words, if SBP is above $199 \mathrm{~mm} \mathrm{Hg}$ it is unquestionably too high (which is labeled High 2 in the Fuzzy set), whereas between 185 and $199 \mathrm{~mm} \mathrm{Hg}$, it could go either way. Same procedure is followed for all the other input variables for determining the fuzzy sets and the membership function. The fuzzy sets form by the classification of each vital sign and the corresponding membership functions are shown below.

Table 2: Classification of Systolic Blood Pressure

\begin{tabular}{|l|l|l|}
\hline Input Field & Range & Fuzzy Sets \\
\hline \multirow{3}{*}{$\begin{array}{l}\text { Systolic Blood } \\
\text { Pressure }\end{array}$} & $<75$ & Low-3 \\
\cline { 2 - 3 } & $70-85$ & Low-2 \\
\cline { 2 - 3 } & $80-100$ & Low-1 \\
\cline { 2 - 3 } & $95-199$ & Normal-0 \\
\cline { 2 - 3 } & $>185$ & High-2 \\
\hline
\end{tabular}

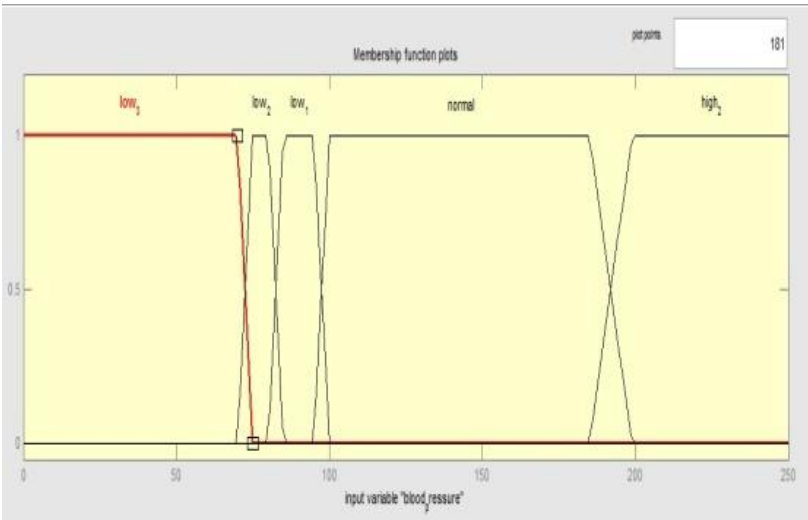

Fig.3: Membership function of systolic blood pressure

Table 3: Classification of Heart Rate

\begin{tabular}{|l|l|l|}
\hline \multirow{4}{*}{ Input Field } & Range & Fuzzy Sets \\
\hline \multirow{5}{*}{ Heart Rate } & $<50$ & Low- 2 \\
\cline { 2 - 3 } & $45-60$ & Low-1 \\
\cline { 2 - 3 } & $53-100$ & Normal \\
\cline { 2 - 3 } & $95-110$ & High -1 \\
\cline { 2 - 3 } & $105-130$ & High-2 \\
\cline { 2 - 3 } & $>125$ & High -3 \\
\hline
\end{tabular}

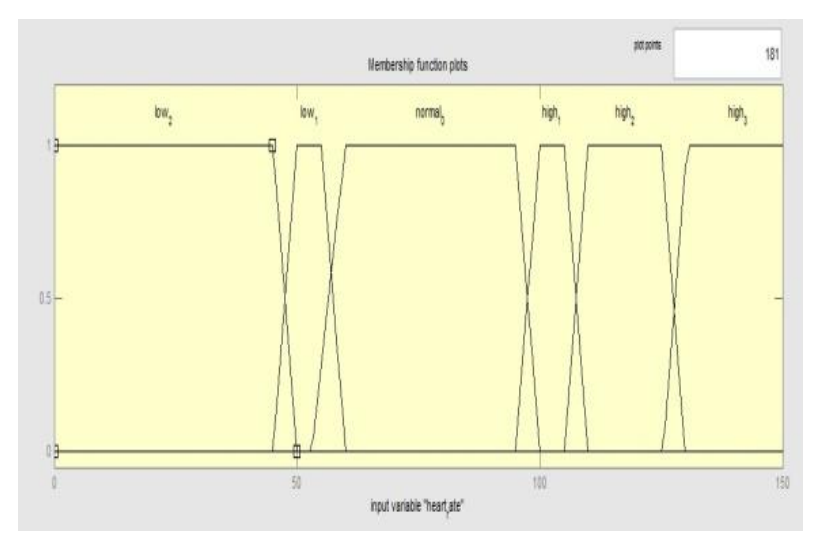

Fig.4: Membership function of Heart Rate

Table 4: Classification of SPO2

\begin{tabular}{|l|l|l|}
\hline Input Field & Range & Fuzzy Sets \\
\hline \multirow{4}{*}{ SPO2 } & $<85$ & Low -3 \\
\cline { 2 - 3 } & $83-90$ & Low-2 \\
\cline { 2 - 3 } & $87-95$ & Low-1 \\
\cline { 2 - 3 } & $>93$ & Normal -0 \\
\hline
\end{tabular}




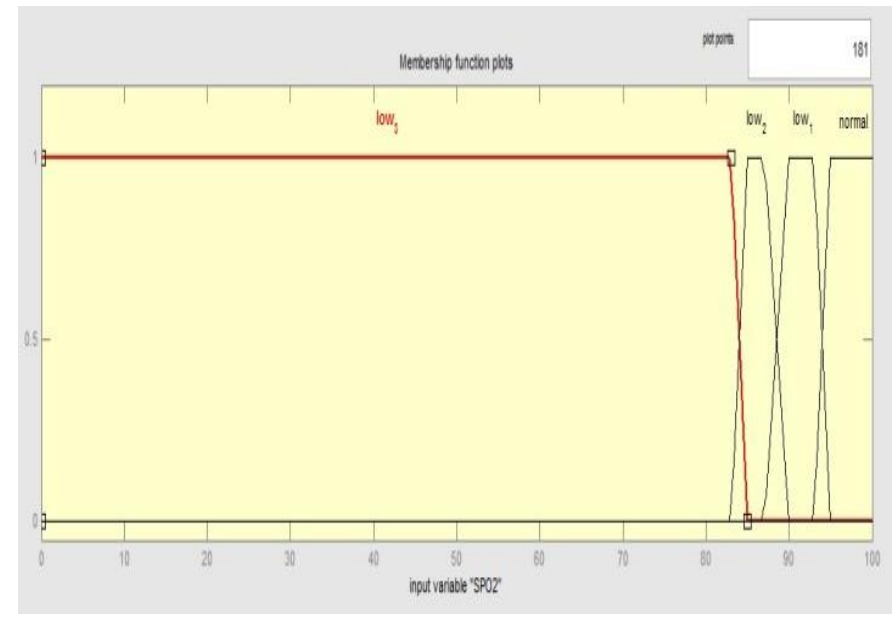

Fig.5: Membership function of SPO2

Table 5: Classification of Temperature

\begin{tabular}{|l|l|l|}
\hline Input Field & Range & Fuzzy Sets \\
\hline \multirow{3}{*}{ Temperature } & $<36.5$ & Low 2 \\
\cline { 2 - 3 } & $36-38.5$ & Normal 0 \\
\cline { 2 - 3 } & $>38$ & High2 \\
\hline
\end{tabular}

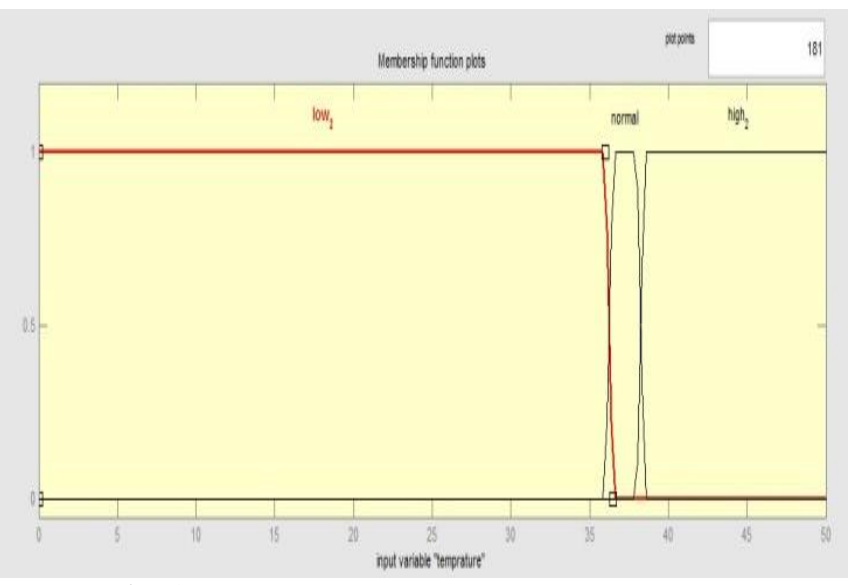

Fig.6: Membership function of Temperature

Table 6: Classification of Blood Sugar

\begin{tabular}{|l|l|l|}
\hline Input Field & Range & Fuzzy Sets \\
\hline \multirow{4}{*}{ Blood Sugar } & $<66$ & Low -3 \\
\cline { 2 - 3 } & $63-72$ & Low-2 \\
\cline { 2 - 3 } & $70-110$ & Normal-0 \\
\cline { 2 - 3 } & $106-150$ & High-2 \\
\cline { 2 - 3 } & $>140$ & High- 3 \\
\hline
\end{tabular}

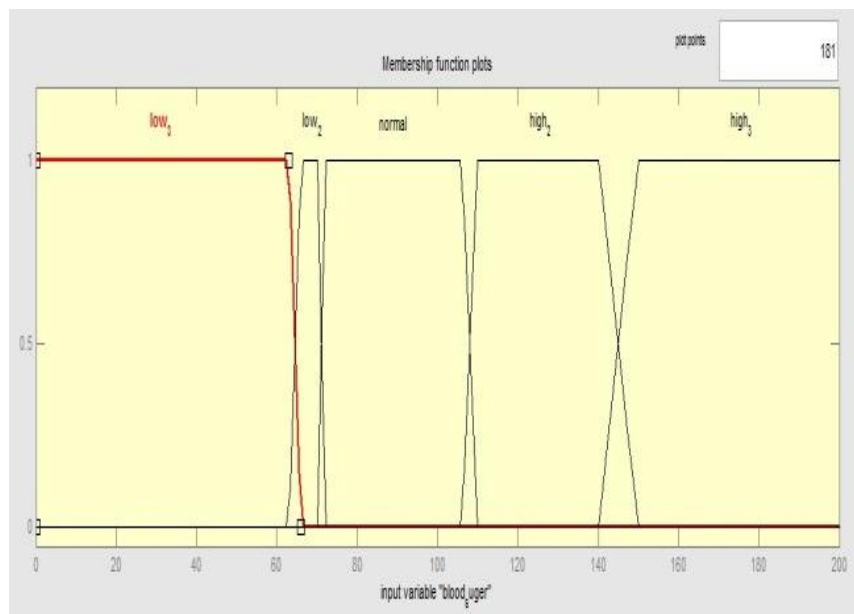

Fig.7: Membership function of blood Sugar

\subsection{Output Variables}

In this fuzzy expert system there is one output variable i.e. the Risk Level, which refers to the degree of illness of the patient. Larger the value of this output variable more will be the health risk of the patient. In this system, we have 15 fuzzy sets for the output variable risk level (NRM, LRG1, LRG2, LRG3, LRG4, HRG5, HRG6, HRG7, HRG8, HRG9, HRG10, HRG11, HRG12, HRG13, and HRG14). Membership functions for these sets are triangular.

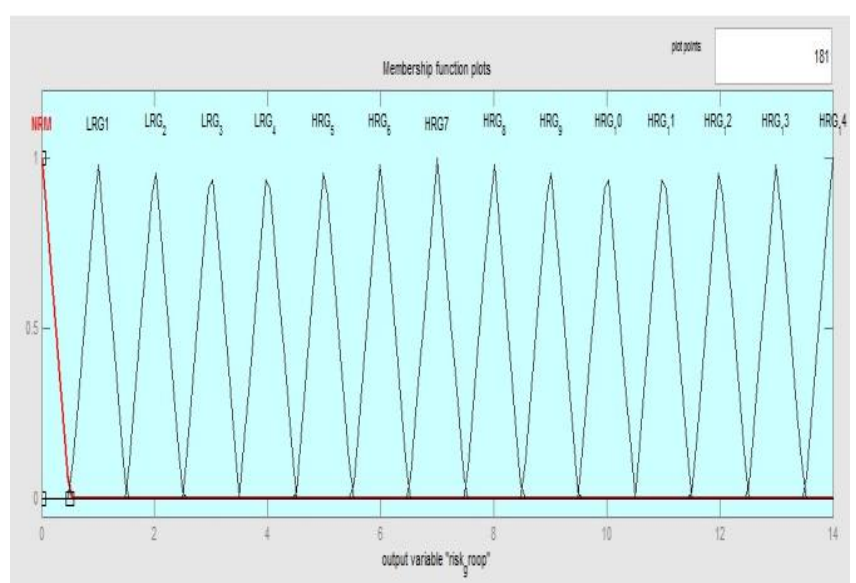

Fig.8: Membership function of Output variable (Risk Level)

Table 7: Classification of Output variable (Risk Level)

\begin{tabular}{|l|l|l|}
\hline $\begin{array}{l}\text { Input } \\
\text { Field }\end{array}$ & Range & Fuzzy Sets \\
\hline \multirow{5}{*}{} & $0<\mathrm{RL}<0.5$ & NRM \\
\cline { 2 - 3 } & $0.5<\mathrm{RL}<1.5$ & LRG1 \\
\cline { 2 - 3 } & $1.5<\mathrm{RL}<2.5$ & LRG2 \\
\cline { 2 - 3 } & $2.5<\mathrm{RL}<3.5$ & LRG3 \\
\cline { 2 - 3 } & $3.5<\mathrm{RL}<4.5$ & LRG4 \\
\cline { 2 - 3 } & $4.5<\mathrm{RL}<5.5$ & HRG 5 \\
\cline { 2 - 3 } & $5.5<\mathrm{RL}<6.5$ & HRG 6 \\
\cline { 2 - 3 } & $6.5<\mathrm{RL}<7.5$ & HRG 7 \\
\hline
\end{tabular}




\begin{tabular}{|l|l|l|}
\hline Risk & $7.5<\mathrm{RL}<8.5$ & HRG 8 \\
\cline { 2 - 3 } Level & $6.5<\mathrm{RL}<9.5$ & HRG 9 \\
\cline { 2 - 3 } & $6.5<\mathrm{RL}<10.5$ & HRG 10 \\
\cline { 2 - 3 } & $6.5<\mathrm{RL}<11.5$ & HRG 11 \\
\cline { 2 - 3 } & $6.5<\mathrm{RL}<12.5$ & HRG 12 \\
\cline { 2 - 3 } & $6.5<\mathrm{RL}<13.5$ & HRG 13 \\
\cline { 2 - 3 } & $13.5<\mathrm{RL}<14$ & HRG 14 \\
\hline
\end{tabular}

\subsection{Fuzzy Rule Base}

The rule base is the main part in the fuzzy inference system and the quality of results in a fuzzy system depends on the fuzzy rules. The designed expert system in this work includes 1800 rules that cover all possible cases. The numbers of rules were obtained using the formula of Equation

$$
\mathrm{N}=\mathrm{I}(1) \times \mathrm{I}(2) \times \mathrm{I}(3) \times \mathrm{I}(4) \times \mathrm{I}(5) \times \mathrm{I}(6) \ldots \ldots \times \mathrm{I}(n)
$$

Where $N$ is the total number of possible rules for a fuzzy system and $I(n)$ is the number of linguistic terms for the input linguistic variable $n$. The rules for this fuzzy expert system were formulated using MEWS scoring system. As all inputs are dependent on each other, therefore in this system we use logical combination of inputs with AND because all the inputs are dependent on each other. The results with the 1800 rules tend to be similar to the MEWS scoring system. A sample of the rules has been shown in Figure 9.

Rule Editor: rpms

File Edit View Options

1. If (blood_pressure is low_3) and (heart_rate is low_2) and (SPO2 is low_3) and (temprature is low_2) and (blood_suger is low_3) then (risk_groop is HRG_14) (1) A

2. If (blood_pressure is low_3) and (heart_rate is low_2) and (SPO2 is low_3) and (temprature is low_2) and (blood_suger is low_2) then (risk_groop is HRG_12) (1)

3. If (blood_pressure is low_3) and (heart_rate is low_2) and (SPO2 is low_3) and (temprature is low_2) and (blood_suger is normal) then (risk_groop is HRG_10) (1)

4. If (blood_pressure is low_3) and (heart_rate is low_2) and (SPO2 is low_3) and (temprature is low_2) and (blood_suger is high_2) then (risk_groop is HRG_12) (1)

5. If (blood_pressure is low_3) and (heart_rate is low_2) and (SPO2 is low_3) and (temprature is low_2) and (blood_suger is high_3) then (risk_groop is HRG_14) (1)

6. If (blood_pressure is low_3) and (heart_rate is low_2) and (SPO2 is low_3) and (temprature is normal) and (blood_suger is low_3) then (risk_groop is HRG_11) (1)

7. If (blood_pressure is low_3) and (heart_rate is low_2) and (SPO2 is low_3) and (temprature is normal) and (blood_suger is low_2) then (risk_groop is HRG_10) (1)

8. If (blood_pressure is low_3) and (heart_rate is low_2) and (SPO2 is low_3) and (temprature is normal) and (blood_suger is normal) then (risk_groop is HRG_9) (1)

9. If (blood oressure is low 3 ) and (heart rate is low 2 ) and (SPO2 is low 3 ) and (temorature is normal) and (blood suoer is hioh 2) then (risk orood is HRG 10 ) (1) - (

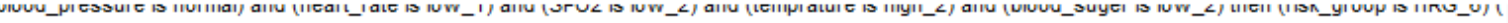
1168. If (blood_pressure is normal) and (heart_rate is low_1) and (SPO2 is low_2) and (temprature is high_2) and (blood_suger is normal) then (risk_groop is LRG_4) (

1169. If (blood_pressure is normal) and (heart_rate is low_1) and (SPO2 is low_2) and (temprature is high_2) and (blood_suger is high_2) then (risk_groop is HRG_6) (

1170. If (blood_pressure is normal) and (heart_rate is low_1) and (SPO2 is low_2) and (temprature is high_2) and (blood_suger is high_3) then (risk_groop is HRG7) (1 1171. If (blood_pressure is normal) and (heart_rate is low_1) and (SPO2 is low_1) and (temprature is low_2) and (blood_suger is low_3) then (risk_groop is HRG_6) (1 1172. If (blood_pressure is normal) and (heart_rate is low_1) and (SPO2 is low_1) and (temprature is low_2) and (blood_suger is low_2) then (risk_groop is HRG_5) (1 1173. If (blood_pressure is normal) and (heart_rate is low_1) and (SPO2 is low_1) and (temprature is low_2) and (blood_suger is normal) then (risk_groop is LRG_3) (C 1174. If (blood_pressure is normal) and (heart_rate is low_1) and (SPO2 is low_1) and (temprature is low_2) and (blood_suger is high_2) then (risk_groop is HRG_5) (

1/Y5. It (blood_pressure is high_2) and (heart_rate is high_3) and (SHO2 is normal) and (temprature is normal) and (blood_suger is high_3) then (risk_groop is HKG_13 1796. If (blood_pressure is high_2) and (heart_rate is high_3) and (SPO2 is normal) and (temprature is high_2) and (blood_suger is low_3) then (risk_groop is HRG_10) 1797. If (blood_pressure is high_2) and (heart_rate is high_3) and (SPO2 is normal) and (temprature is high_2) and (blood_suger is low_2) then (risk_groop is HRG_13) 1798. If (blood_pressure is high_2) and (heart_rate is high_3) and (SPO2 is normal) and (temprature is high_2) and (blood_suger is normal) then (risk_groop is HRG_8) 1799. If (blood_pressure is high_2) and (heart_rate is high_3) and (SPO2 is normal) and (temprature is high_2) and (blood_suger is high_2) then (risk_groop is HRG_13 1800. If (blood_pressure is high_2) and (heart_rate is high_3) and (SPO2 is normal) and (temprature is high_2) and (blood_suger is high_3) then (risk_groop is HRG_10 1

Fig.9: Sample of the Fuzzy Logic System Rules

\subsection{Fuzzification and Defuzzification}

Fuzzification is the first step in the design of any fuzzy expert system. It is the process of mapping a crisp value of an input to membership degrees in different Fuzzy Linguistic variables.. Defuzzification is the inverse process of fuzzification. It is the process of combining fuzzy output of all the rules to give one crisp value. Thus crisp value output is given by the defuzzification process after estimating its input value. An example of the designed system results in MATLAB is shown in Figure 10.

The following values are given to each input field: Systolic Blood Pressure $(\mathrm{SBP})=120$, Heart Rate $(\mathrm{HR})=75$, SPO2=98, Temperature $($ TEMP) $=37$ and Blood Sugar $(B S)$ $=95$. The fuzzy logic engine is triggered. The MATLABrule viewer and simulation results are shown in Figure10
.New input values generate new depression risk output responses.

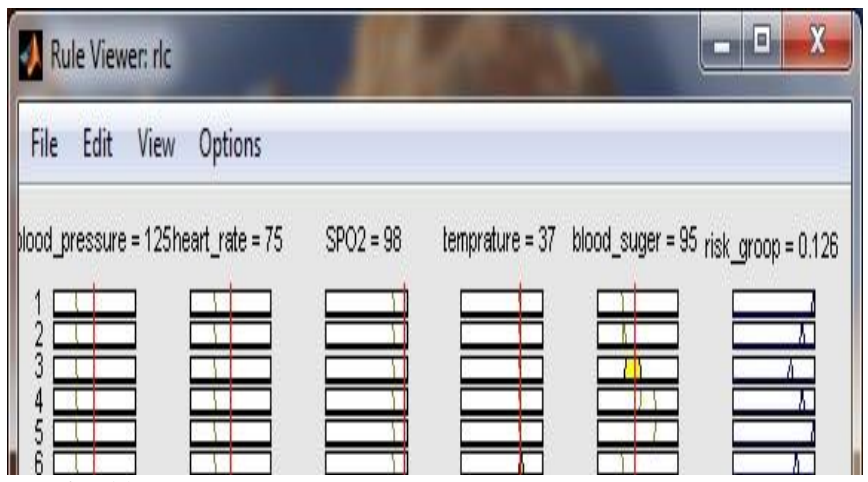

Fig.10: Rule viewer showing a simulation result of the designed system 
Surface viewer of some fields as follow

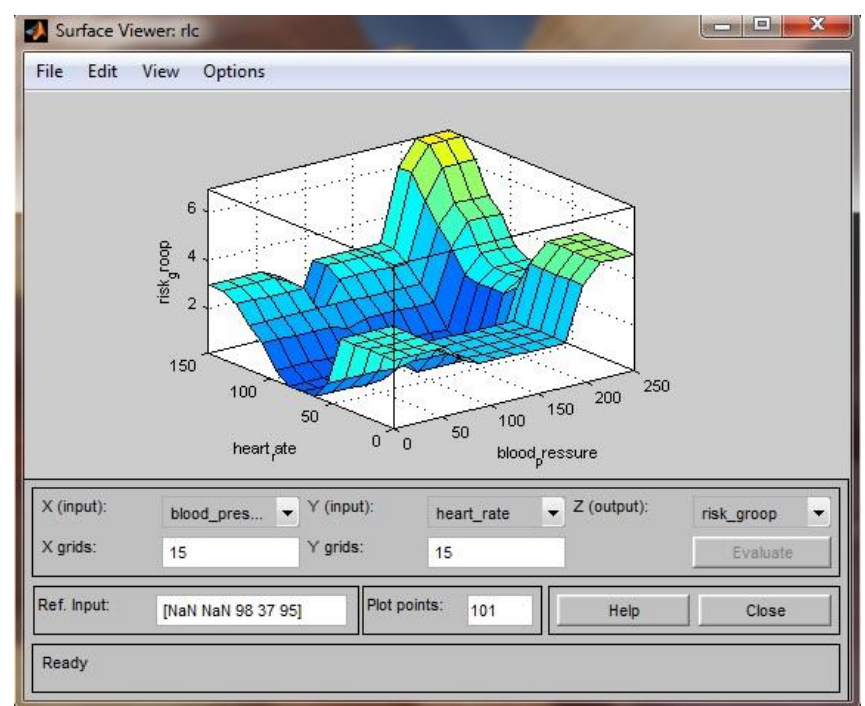

Fig.11. Surface Viewer of Blood Sugar and Heart Rate

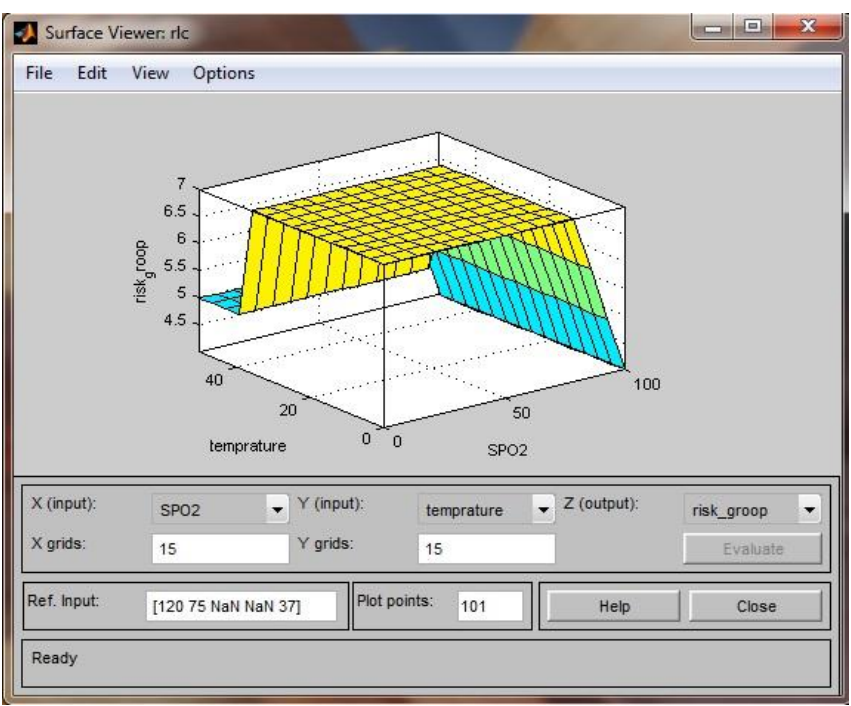

Fig.12. Surface Viewer of temperature and SPO2

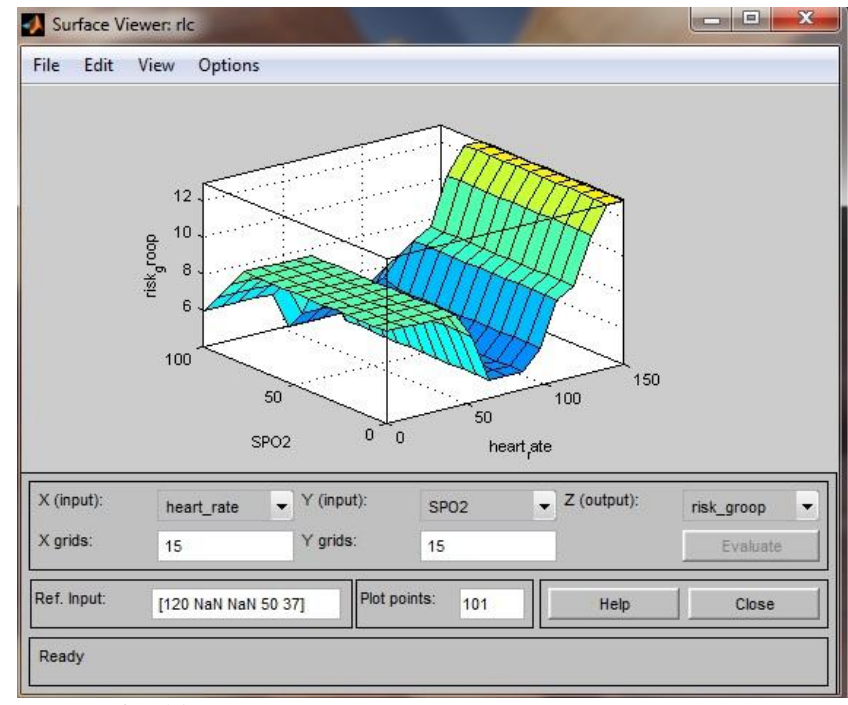

Fig.13. Surface Viewer of SPO2 and Heart Rate

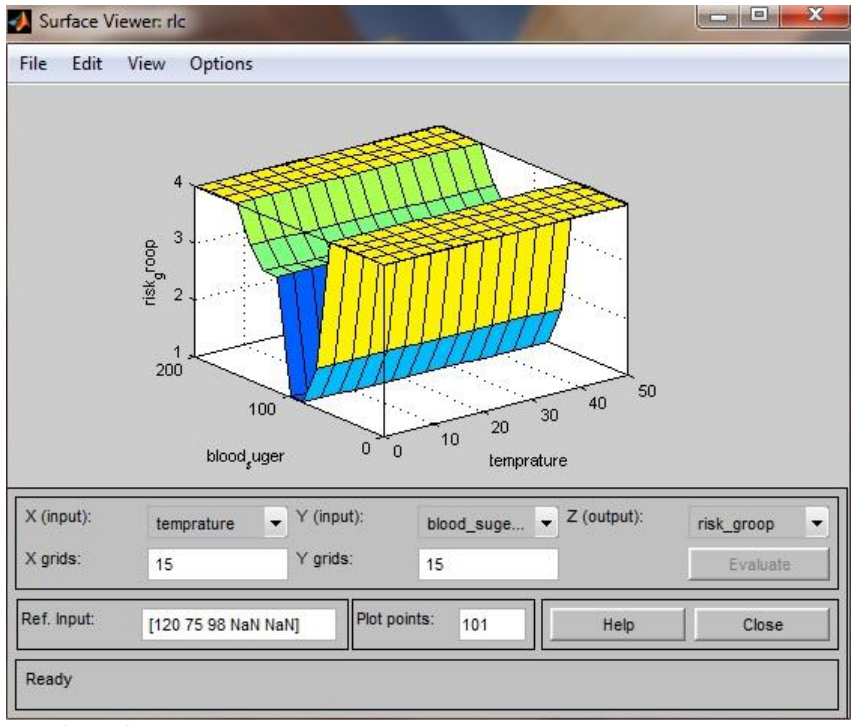

Fig.14. Surface Viewer of temperature and blood sugar

\section{RESULT AND DISCUSSION}

The designed expert system has been tested with some set of values of patient's vital signs as shown in Table 8.. Also a comparison between the MEWS scoring system results and fuzzy logic results has been done in order to evaluate the performance of the designed system.

Now in Table 8, total 6 cases are considered. Case 1 and Case 2 corresponds to normal conditions of the patient. For these two cases score according to MEWS system is 0 while the designed expert system gives a score of .126 for each case which is also very close to 0 and hence the result is quite satisfactory. Again Case 3 and Case 4 corresponds conditions when patient is under low risk. The score according to MEWS system for Case 3 is 2 and that for Case 4 is 3 . For these two cases the designed expert system gives a score of 2 and 3. Similarly Case 5 and Case 5 correspond when the patient is under high risk. For these two cases also the result of the designed system is satisfactory. 
Table 8: A comparison between the mews results and the fuzzy logic results

\begin{tabular}{|c|c|c|c|c|c|c|c|c|c|}
\hline \multirow[t]{3}{*}{ S1 No } & \multirow{2}{*}{\multicolumn{5}{|c|}{ Vital Signs }} & \multicolumn{4}{|c|}{ Result } \\
\hline & & & & & & \multicolumn{2}{|c|}{ Using MEWS } & \multicolumn{2}{|c|}{ Using Fuzzy Logic } \\
\hline & SBP & HR & SPO2 & TEMP & $\mathrm{BS}$ & Status & Score & Status & Score \\
\hline 1 & 120 & 65 & 95 & 37 & 88 & NRM & 0 & NRM & .126 \\
\hline 2 & 170 & 80 & 96 & 37.5 & 102 & NRM & 0 & NRM & .126 \\
\hline 3 & 117 & 63 & 98 & 38.2 & 115 & LRL & 2 & LRL & 2.42 \\
\hline 4 & 180 & 92 & 97 & 37 & 220 & LRL & 3 & LRL & 3 \\
\hline 5 & 187 & 87 & 97 & 41 & 161 & HRL & 5 & HRL & 4.67 \\
\hline 6 & 190 & 120 & 94 & 38.6 & 120 & HRL & 6 & HRL & 6.82 \\
\hline
\end{tabular}

\section{CONCLUSION}

The paper describes a design of a fuzzy expert system for determination of the risk level of patient, which can be used in any situation when it is necessary to predict the health status of patient. The designed system can be used by the doctor or by the patient himself. In this paper it can be concluded that using expert knowledge embedded as fuzzy rules and supplied input data (i.e. patent's vital signs), the designed system predicts risk level of patient and it can be easily verified by the comparison done in table 9 . Finally it can be concluded that up to some extent the designed system can be used as a virtual doctor.

\section{REFERENCES}

[1]. M. Mayilvaganan, K. Rajeswari, "Risk Factor Analysis to Patient Based on Fuzzy Logic Control System" , International Journal of Engineering Research and General Science Volume 2, Issue 5, August-September, 2014 ISSN $2091-2730$

[2]. The Free Encyclopedia $\quad$ From Wikipedia."Http://En.Wikipedia.Org/Wiki/Mews,"

[3]. Mansoor Mirza, Hamid GholamHosseini, Member, IEEE, Michael J. Harrison, "A Fuzzy Logic-based System for AnaesthesiaMonitoring", 32nd Annual International Conference of the IEEE EMBS Buenos Aires, Argentina, August 31 - September 4, 2010

[4]. M. Mayilvaganan, K.Rajeswari, "Health Care Analysis Based On Fuzzy Logic Control System", International Journal of Computer Science Trends and Technology (IJCST), Volume 2 Issue 4, Jul-Aug 2014

[5]. Smita Sushil Sikchi, Sushil Sikchi, Ali M. S, "Generic Medical Fuzzy Expert System for Diagnosis of Cardiac Diseases" , International Journal of Computer Applications (0975 - 8887) Volume 66-No.13, March 2013

[6]. Adult Modified Early Warning Score (MEWS) Policy and Escalation Pathway Version 3

[7]. MultiCare Health System: Using a Modified Early Warning System (MEWS) to Improve Patient Safety HIMSS InnovationCommunity November 2, 2012

[8]. Ali.Adeli, Mehdi.Neshat, "A Fuzzy Expert System for Heart Disease Diagnosis", Proceeding of international Multiconference of Engineers and Computer Scientists 2010 Vol I,IMECS 2010,March 17-19,2010,Hong Kong

[9]. L.A. Zadeh,"fuzzy theory," university of California,Berkely, 1965
[10]. "Understanding Fuzzy Logic: An Interview With Lotfi Zadeh," IEE Signal Processing Magazine, May 2007.

[11]. Paul Grant, "A New Approach To Diabetic Control: Fuzzy Logic And Insulin Pump Technology," Department Of Medicine, Royal Sussex County Hospitall,Medical Engineering \& Physics, 2007.

[12]. Smita Sushil Sikchi, Sushil Sikchi, Ali M. S. "Fuzzy Expert Systems (FES) for Medical Diagnosis", International Journal of Computer Applications (0975 8887) Volume 63- No.11, February 2013

[13]. L. Zadeh, "Fuzzy Sets," Information And Control, Vol. 8, No. 3, 1965 